\title{
Entrelacs
}

ENTRELACS Cinéma et audiovisuel

10 | 2013

Le Toucher

\section{Lady Chatterley - du roman au film : la reconquête du corps par le toucher}

\section{Rémi Gonzalez}

\section{(2) OpenEdition}

Journals

Édition électronique

URL : http://journals.openedition.org/entrelacs/534

DOI : $10.4000 /$ entrelacs.534

ISSN : 2261-5482

Éditeur

Éditions Téraèdre

Référence électronique

Rémi Gonzalez, "Lady Chatterley - du roman au film : la reconquête du corps par le toucher », Entrelacs [En ligne], 10 | 2013, mis en ligne le 12 septembre 2013, consulté le 19 avril 2019. URL: http://journals.openedition.org/entrelacs/534 ; DOI : 10.4000/entrelacs.534

Ce document a été généré automatiquement le 19 avril 2019

Tous droits réservés 


\title{
Lady Chatterley - du roman au film : la reconquête du corps par le toucher
}

\author{
Rémi Gonzalez
}

La problématique du toucher a toutes les chances de devenir centrale dans le cadre d'une œuvre ayant partie liée avec l'érotisme, à tout le moins dont le sujet principal s'axe autour de la question du corps et de l'éveil des sens. C'est précisément ce qui est en jeu dans The Second Lady Chatterley's Lover ${ }^{1}$ de David Herbert Lawrence (écrit en 1927 et publié pour la première fois en 1972) et, par conséquent, dans son adaptation cinématographique réalisée par Pascale Ferran en 2006, Lady Chatterley². Le roman de Lawrence raconte la réanimation du corps de son héroïne, châtelaine mariée à un invalide de guerre, et sa redécouverte du plaisir à travers une histoire d'amour adultérine avec le garde-chasse du domaine de son mari. The Second Lady Chatterley's Lover, s'il ne se départit pas d'une forte attention portée à l'intériorité des personnages, est en dernière analyse le roman d'un épanouissement corporel, d'une réincarnation de la chair. Le romancier, comme la cinéaste, traitent ce lent apprentissage du contact physique en convoquant une expérience tactile du monde, qui passe par la découverte de l'autre via le toucher : « Her soul would have to have some relief, some hope, some touch. It was that she wanted. Not any revelation nor any new idea. A new touch. Just a touch. $»^{3}$

D'une œuvre à l'autre, percent la connivence artistique entre deux personnalités et la volonté de Pascale Ferran de prolonger le mouvement inauguré par Lawrence. Ainsi, à une note de Lawrence, très représentative de la conception que l'écrivain se faisait de la littérature - «Je pense que l'art doit révéler l'instant dans sa palpitation. $»^{4}$ - pourrait répondre celle de la cinéaste :

J'essaie toujours d'être au plus près de la présence du monde : tenter de capter les frémissements du vent, presque les odeurs, si je pouvais... Et, dans Lady Chatterley, c'est enrichi de sensations tactiles [...]..$^{5}$

Nous nous proposons donc, dans une étude comparative, d'analyser les moyens littéraires et cinématographiques voués à créer les conditions d'une lente attraction des corps tendue vers le toucher. Mais ce simple toucher, vital à l'héroïne du récit, met un certain 
temps à se réaliser et passe par d'autres formes de contact, à commencer par la vision. Évoquant une « vision haptique » telle que définie par Gilles Deleuze ${ }^{6}$, le regard va se faire l'agent du contact entre deux êtres et rompre les lois cartésiennes de l'espace pour invoquer la figure de l'autre et la rendre quasi-palpable. Va ensuite s'opérer un déplacement vers un toucher absent, quand le contact sera transféré sur des objets vivants ou portant trace du vivant, dans un rapprochement où la figure de la main tiendra une place essentielle - autant de passages obligés avant que l'étreinte espérée ne soit enfin célébrée.

L'histoire étant celle d'une révolution totale de l'être par le corps, il faut que l'héroïne défaille avant que de renaître à soi, d'où une première partie (dans le roman et dans le film) vouée à faire état d'une déperdition morale et physique, du dépérissement progressif de la jeune Constance, corps inanimé, exsangue voire absent. Après quoi, petit à petit, plusieurs événements vont conduire au sacro-saint toucher salvateur. Le premier contact physique entre Constance et Parkin, le garde-chasse de Wragby, ne survient qu'à la page 105 du roman et aux alentours de la 45ème minute du film. Mais ce contact n'est pas sans précédents, à commencer par celui du regard de la femme posé sur le corps de l'homme. Or cette première étape vers le toucher est aussi une première rencontre qui va se doubler dans le texte et à l'écran d'une pure épiphanie, où la « vision haptique » tend à actualiser le corps de l'autre.

\section{L'épreuve du premier regard}

Constance est envoyée un matin à la rencontre du garde-chasse pour lui faire une commission. Si l'héroïne a déjà croisé Parkin à deux reprises dans le roman, de façon toujours brève et tendue, c'est en revanche leur première rencontre dans le film, mais ici et là le choc est le même. C'est celui d'une mort intérieure au bénéfice d'une renaissance imminente. La violence du contact est suggérée par une métaphore très nette dans le texte: "She was so sure that there was nobody, that she came suddenly into the little open gateway of the yard, and there stopped as if she had been shot. $»^{7}$ Dans le film, Constance (Marina Hands) va frapper à la porte du garde-chasse mais n'obtient aucune réponse. Elle se hisse sur la pointe des pieds pour jeter un œil à l'intérieur à travers une lucarne, et Pascale Ferran réalise alors le premier gros plan du film sur son héroïne, ou plus précisément sur les bottines de son héroïne, isolant une partie du corps de Constance ainsi mise en valeur dans un effort patent comme pour signifier que ce corps a une chance d'exister en dehors du château, en un lieu nouveau, non-adapté à la vie bien rangée des femmes du monde et réclamant d'elle quelque vitalité. Puis Constance longe le côté de la maisonnette, filmée de face, en plan rapproché et travelling arrière, marchant, insouciante, les yeux fixés sur les poules devant elle. Parvenant au coin de la maison, la jeune femme tourne sur sa gauche vers l'espace enfin ouvert dans la partie droite du cadre, mais se trouve soudain interdite, immobilisée, heurtée par une vision insoupçonnée. Filmée de profil au moment où elle tourne à l'angle de la maison, le léger mouvement de recul de Constance en impose un à la caméra qui reste sur la jeune femme et maintient pour un temps l'objet de sa vision dans le hors-champ. Un plan d'ensemble en vue subjective nous laisse alors découvrir Parkin (Jean-Louis Coulloc'h), en pleines ablutions, de dos, torse nu, le pantalon rabattu sur les hanches, penché sur un tonneau, s'aspergeant vigoureusement la tête et la nuque. Après cette première apparition du garde-chasse, on revient très vite sur Constance, cadrée de face et en gros plan, depuis le 
point de vue qui serait celui de l'homme si par malheur il venait à se retourner. La jeune femme observe le corps qui se dresse devant elle encore quelques secondes, effarée, puis recule dans le plan sans faire de bruit. Après un cut brutal (on passe d'un plan fixe à une caméra portée en travelling), Constance fuit en courant cette vision interdite.

\section{Le corps chez Lawrence : entre apparition et incarnation}

Vient alors dans le roman une longue résurgence de l'image du garde-chasse, aussi étale que la vision en elle-même et sa première description, très factuelle pour ne pas dire objective, furent brèves. Sur plus d'une page, l'écrivain déroule le flux de conscience de son héroïne, manifestement bouleversée par l'émotion née d'un tel choc visuel :

The white torso of the man had seemed so beautiful to her, opening on the gloom. The white, firm, divine body, with its silky ripple, the white arch of life, as it bent forward over the water, seemed, she could not help it, of the world of gods. There still was a world that gleamed pure and with power, where the silky firm skin of the man's body glistened broad upon the dull afternoon. [....] She had seen beauty, and beauty alive. That body was of the world of the gods, cleaving through the gloom like a revelation. ${ }^{8}$

Lawrence use admirablement ici de ce qui caractérise en propre son style : une écriture poétique aux tons liturgiques, amalgamant les évocations du sacré aux réalités prosaïques du monde dans une volonté résolument païenne d'en référer à toutes les croyances afin d'invoquer l'avènement de la matière par des répétitions hypnotiques. Le texte, à cet endroit, et c'est une habitude pour Lawrence, est littéralement saturé de références mythologiques. La scène invite notamment à être lue comme une réécriture du mythe d'Actéon et Diane dans Les Métamorphoses d'Ovide, mythe du désir voyeuriste que Lawrence plie à son souhait d'un rééquilibre des rôles en matière d'amour, faisant de la Déesse un Dieu ${ }^{9}$ et du simple mortel une femme tétanisée de désir. L'écrivain densifie ce mythe détourné via l'ajout de plusieurs épaisseurs référentielles supplémentaires en faisant directement allusion à d'autres topoï partagés par l'inconscient collectif, et notamment religieux, avec les références à l'Arche de Noé ou à l'Arche d'Alliance de la Bible, achevant de déifier la figure du garde-chasse. Mais Lawrence ne retient des textes sacrés que leur potentiel d'évocation, leur poésie intrinsèque - celle de la prière fervente et de l'extase pure - pour les délester de leur fond moral ou de toute interprétation idéologique. Et l'écrivain poursuit immédiatement dans le même souffle :

A great soothing came over her heart, along with the feeling of worship. The sudden sense of pure beauty, beauty that was active and alive, had put worship in her heart again. Not that she worshipped the man, nor his body. But worship had come into her, because she had seen a pure loveliness, that was alive, and that had touched the quick in her. It was as if she had touched God, and been restored to life. The broad, gleaming whiteness $!^{10}$

Dans cet extrait et dans le précédent, les nombreuses hyperbates donnent un relief particulier aux phrases et les font rejaillir. Les termes sont inlassablement répétés, les polyptotes et les assonances sont légion, de sorte que par un long procédé d'accumulation, Lawrence se livre à un vibrant exercice d'hypotypose, donnant à lire une description qui, pour peu réaliste qu'elle soit, s'avère pour le moins animée et vivante. La scène, ainsi imagée, est rendue présente aux yeux du lecteur et semble être dite à l'instant même où elle est vécue. Les termes «body» et «skin» sont associés à ces mots 
qui invoquent la présence des choses et les font affleurer : «opening ", «revelation». De sorte qu'en suggérant diverses alliances antithétiques dans un système de dualités, à l'image des thèmes de l'ombre (« gloom ») et de la lumière (« white »), ou du terrestre et du divin, Lawrence s'obstine à faire apparaître comme par miracle le corps incarné de Parkin au lecteur, par une description frappante qui a tout de la répétition litanique répétitive et embrasse le ton exalté de la poésie dans ce qui s'apparente au fond à une pure prose lyrique.

Dans ce passage ô combien mystique, c'est bel et bien un instant d'incarnation qui s'offre au lecteur. Constance entrevoit le divin, immergé dans les ténèbres d'un sombre décor, qui cependant illumine, irradie les alentours de sa lumière immanente en s'incarnant miraculeusement par une apparition charnelle ferme ("firm ») et ondoyante ("ripple ») à la fois. De sorte que le corps de Parkin est simultanément dématérialisé dans son accession à l'ordre divin, et ramené à une présence ostentatoire, tandis que Constance évoque sa peau et ses muscles et les fait exister par les mots. C'est ce mariage surprenant entre une réalité palpable, visible, épidermique, existante et son apparition blanche, ondoyante, divine, miraculeuse et mystique, qui fait toute la force de cette scène et insuffle sa puissance résurrectionnelle à la vision de Constance en même temps qu'elle dit la puissance d'incarnation du regard. Le contact des corps, bien que possible ( He was so near, Constance could have touched him $»^{11}$, écrit Lawrence juste avant que son héroïne ne s'enfuie dans le bois), n'a pas eu lieu, mais un contact visuel s'y est substitué qui a pris la force d'un pur contact physique. C'est ce que Lawrence signifie par les nombreuses occurrences du mot «touched» et en décrivant le corps de Parkin en des termes tantôt mystiques, qui disent bien le caractère intangible, fantomatique pour ne pas dire fantasmatique, de l'apparition, tantôt en des termes beaucoup plus réalistes, qui mettent l'accent sur la fermeté de cette image, ses reliefs, sa matière, et qui, couplés à une litanie répétitive invoquant la présence du corps, finissent par le faire surgir du texte, sous les yeux, et même sous les doigts, de Constance. Ayant fui dans le bois, l'héroïne doit se contenter d'une image qui, dans une prière extatique, tend vers une "vision haptique " deleuzienne à force d'évocation et de ressassement, surgissant de nulle part, miraculeusement matérialisée comme s'il fallait que «l'image [...] cesse d'être imageaction pour devenir une image optique, sonore (et tactile) pure. $»^{12}$

\section{Faux-raccord cinématographique et vision à distance}

Dans le film de Pascale Ferran, Constance, réfugiée dans la forêt et assise sur un banc, n'a que le temps de pousser un grand soupir pour se rétablir quand l'image du dos de Parkin surgit à nouveau dans un faux-raccord surprenant: le garde-chasse apparaît soudainement, comme un contrechamp impossible au regard de l'héroïne réfugiée dans la forêt, assise et fixant un point devant elle, comme assaillie de visions incontrôlables auxquelles elle semble réagir par un léger soubresaut. Car Parkin ne peut être vu de Constance, qui ne fait qu'évoquer en pensées ce dos blanc qu'elle n'a plus sous les yeux, mais dont l'image vient néanmoins s'insérer dans le cadre spatio-temporel de la jeune femme. Le texte est formel : "She withdrew in haste and in silence, back to the wood. There, she hastened through the wet trees, to be safely out of sight of the cottage. ${ }^{13}$ Du reste, l'image de Parkin intégrée par le montage n'est pas exactement celle que nous avons découverte précédemment avec Constance. C'est un autre plan, nouveau et d'autant plus impossible que la taille du cadre a changé : de large il est devenu très rapproché, fétichisant ce dos 
qui occupe désormais presque toute l'image, alors que Constance n'a manifestement pas pu le voir d'aussi près. Le même plan se répète ainsi de manière altérée, dans ce que l'on nommera pour l'occasion un polyptote cinématographique. Puis une deuxième apparition résurgente a lieu, interrompant de nouveau le plan sur Constance pour faire surgir une nouvelle image de Parkin, encore sensiblement différente puisque le corps de l'homme est désormais cadré en plan américain. Or non seulement les images sont autres, mais elles ne constituent pas de simples variations sur une même action: Parkin continue sa toilette à l'image, au-delà de ce que Constance a véritablement pu voir. ${ }^{14}$ Nous ne pouvons donc définitivement pas parler d'un flash-back, même si le montage relie les plans par un regard dans le vide de Constance, qui n'est pas sans faire écho chez le spectateur à un certain cliché du personnage abandonné à un ressouvenir inconscient. En réalité il s'agit bel et bien de plans supplémentaires, de pures visions, en dépit d'une incohérence spatiale sidérante. Bachelard écrit :

L'émerveillement est une rêverie instantanée. $»^{15}$ Cet émerveillement bouleverse Constance, qui se retrouve, pour reprendre les mots de Gilles Deleuze, face à «[...] quelque chose de trop puissant, [...] mais parfois aussi de trop beau [...] une beauté trop grande pour nous, comme une douleur trop forte. ${ }^{16}$

La jeune femme est ébranlée au point que le film génère à travers elle de nouvelles images, inédites et impossibles :

Le personnage est devenu une sorte de spectateur. Il a beau bouger, courir, s'agiter, la situation dans laquelle il est déborde de toutes parts ses capacités motrices, et lui fait voir et entendre ce qui n'est plus justiciable en droit d'une réponse ou d'une action. Il enregistre plus qu'il ne réagit. Il est livré à une vision, poursuivi par elle ou la poursuivant, plutôt qu'engagé dans une action. ${ }^{17}$

Constance, figée sur place, est en effet poursuivie par sa vision, vision à distance qui se continue malgré elle et en dépit de toute logique. L'objet lointain est importé dans le présent immédiat du sujet émerveillé via une réduction improbable de l'espace où le corps est à portée de main.

Par un travail de montage, ces insertions hallucinées de l'image de Parkin répondent aux multiples répétitions du texte de Lawrence aspirant à l'avènement du corps présent par un effet de pulsation littéraire et de friction sonore, un " battement nu surgissant du moment instantané. $\aleph^{18}$ Toutefois, si elle préserve la nature chimérique de l'apparition du corps de Parkin, Pascale Ferran diminue de beaucoup la dimension mystique de la vision en elle-même, préservant au dos du garde-chasse son aspect obstinément humain. Aucune tentative de la part de la cinéaste de transposer par un travail spécifique sur la photographie ou par un quelconque effet spécial la " resplendissante blancheur » du corps divinisé de Parkin. Trois longs paragraphes du roman sont condensés dans deux plans lorsque le corps de l'homme est non seulement incarné mais apparaît en toute irréalité par la grâce d'un faux-raccord. Et notre regard d'être endigué par la mise en scène, qui nous place précisément du point de vue de Constance - nous sommes dans sa tête - tout en variant les échelles de plans pour révéler la fantasmagorie. Ces valeurs de plans disent la puissance tactile du regard de la jeune femme, quand la première apparition fait resurgir le dos de Parkin en plan rapproché avant que le second flash ne l'éloigne, comme si la vision rapprochait temporairement Constance de ce toucher inespéré et la portait aux confins du regard, où le contact physique est frôlé. On quitte alors un instant le visage hagard de Constance pour un gros plan sur ses mains relâchées, oubliées sur ses cuisses, paumes tournées vers le haut, ces mains qui n'ont rien touché mais qui sont comme toutes retournées d'avoir été si proche d'un corps. En découvrant 
pour la première fois et par effraction ce corps d'homme, synonyme d'un nouveau monde insoupçonné, Constance découvre la possibilité du corps humain dans son entier, et du sien en particulier.

\section{Le toucher absent}

La séquence de la toilette a un statut de scène matricielle et trouve des échos à différents stades de l'histoire, qui marquent autant d'étapes dans l'évolution de Constance vers une renaissance. Cette scène primitive installe une dialectique du désir, pris entre absence et présence. Pour Pascal Quignard :

Cicéron a défini le désir [...] : le désir est la libido de voir quelqu'un qui n'est pas là. La désidération est la joie de voir l'absent [...] Le désir est l'appétit de voir absent.

L'art voit absent. La jeune fille "voit absent" celui qu'elle aime. ${ }^{19}$

Dans la séquence où elle découvre le corps de Parkin, Constance est prise de " désidération ", cet appétit de l'objet aimé - précisément aimé pour son absence - qui le donne à voir et le rend paradoxalement présent. Pour parer à cette absence, à cet inaccessible, des objets de substitution sont convoqués. Une scène, qui vient faire écho à la séquence de la toilette de Parkin, donne lieu à un tel transfert, lorsque Constance s'émancipe des murs froids du château pour retourner voir du côté de la maison du garde-chasse, où les premières jonquilles viennent d'éclore. Dans le roman comme dans le film, la scénographie et les déplacements du personnage sont les mêmes que dans la scène de la toilette. Constance, essoufflée par sa promenade vers la maison du gardechasse, s'assied un instant sur un banc, les mains posées sur les cuisses, reprenant la position dans laquelle elle s'était arrêtée après avoir vu pour la première fois Parkin. Puis au détour d'un virage, elle est soudain stoppée dans sa marche par la vision d'un champ de jonquilles, qui a littéralement remplacé Parkin, confondu tout au long du roman avec la forêt. Elle s'immobilise et observe avec surprise un parterre de fleurs jaunes que nous découvrons depuis son point de vue, en caméra subjective, comme nous avions découvert Parkin. Mais cette fois-ci la jeune femme descend la pente du talus vers le pré fleuri en accélérant le pas, et la caméra de la filmer de face, la suivant cahin-caha dans cette brève course en avant. La fuite à reculons de la séquence de la toilette est ainsi contredite, tandis que Constance se propulse vers l'objet de son désir. Elle se fige de nouveau dans la contemplation de ce paysage plein de vitalité. Gros plan sur sa main droite, relâchée, abandonnée, et nouveau gros plan sur le visage béat d'admiration de la jeune femme, bouche entrouverte et déjà parée d'un semblant de sourire. Puis à la faveur d'une ellipse nous découvrons un plan serré sur la main de Constance marchant dans la forêt, qui tient un bouquet de jonquilles. Si, dans cette scène, Lawrence met l'accent sur une analogie visuelle entre Parkin et les fleurs par toute une description basée sur le champ sémantique de la lumière, Pascale Ferran, par les gros plans, concentre quant à elle ses effets sur l'idée même du toucher en tant qu'il est absent. Nous ne l'avons pas vu mais un premier contact a néanmoins eu lieu. Constance a touché le corps du garde-chasse par procuration en touchant le monde végétal qui lui correspond. Le toucher a disparu dans le raccord ${ }^{20}$ pour devenir pur toucher absent, annonçant l'avenir tactile des corps au présent. 


\section{Contacts de substitution}

Lawrence - et Pascale Ferran à sa suite - décline les avatars d'un contact repoussé, différé. Mais il s'agit maintenant pour Constance d'aspirer à un véritable contact, ferme et décisif, avec le monde de Parkin :

But with quietness, with an abandon of self-assertion and a fulness of the deep, true self one can approach another human being, and know the delicate best of life, the touch. The touch of the feet on the earth, the touch of the fingers on a tree, on a creature, the touch of hands and breasts, the touch of the whole body to body, and the interpenetration of passionate love : it is life itself, and in the touch, we are alive. $^{21}$

Ce contact intervient dans le roman lorsque Constance, dans la forêt, se met à l'ombre d'un arbre puis s'adosse à son tronc. Lawrence fait alors appel à l'un des procédés littéraires fondamentaux de son écriture, le recours au symbole, en assimilant l'arbre au corps de Parkin : "[...] a young pine-tree, that swayed against her like en animate creature, so subtly rubbing itself against her, the great, alive thing with its top in the wind! ${1^{22}}^{2}$ Dans le film, Pascale Ferran symbolise l'attente désespérée d'un contact par un gros plan sur la main de Constance qui touche tendrement l'écorce de l'arbre recouverte de mousse dans un geste proche de la caresse érotique. Et, ainsi filmé en gros plan, le végétal, épais, humide, semble modeler la surface filmique de sa texture même, nous faisant éprouver visuellement le relief que frôle le personnage. Après le contact des doigts de Constance sur l'arbre, la nécessité évoquée par Lawrence d'un contact des pieds sur le sol est à son tour réalisée à l'image, mise en acte, quand Constance grimpe vivement sur le rondin d'un pont de bois qui passe au-dessus d'une source dans la forêt. Filmé en gros plan, le pas de la jeune femme sur le ponton est si franc qu'il fait tanguer le tronçon de bois, et la caméra par la même occasion, rendant tangible un contact plein et entier. Puis Constance s'apprête à boire à la source, et «négligeant la tasse accrochée à son clou, elle plonge ses mains dans l'eau glacée et boit dans ses mains en coupe. ${ }^{23}$ S'émancipant enfin vis-à-vis des bonnes manières que lui impose son rang, la nouveauté du contact des mains de Constance avec l'eau vivifiante du flux de la source est d'autant plus frappante que cette courte scène intervient comme une variation. En effet, plus tôt dans le film, on a vu Constance emprunter le même pont de bois avec une précaution presque aérienne, quasiment sur la pointe des pieds, filmée en plan large et en plongée, de même qu'on l'a déjà vue boire l'eau de la source du bout des lèvres et dans la petite tasse prévue à cet effet. La séquence est donc rejouée à l'identique à ceci près que chaque contact est désormais filmé en gros plan pour en révéler la force : les pas de Constance sont décidés, elle pénètre dans le sol comme elle plonge profondément ses mains dans l'eau du ruisseau.

\section{De la vision tactile à l'étreinte physique}

Ces contacts répétés avec le corps de la forêt valent pour promesse d'un contact inespéré avec le corps de Parkin et sont autant de pas vers la cabane où l'étreinte aura lieu. Lawrence conjugue le besoin vital d'un contact avec celui du désir charnel en nous ouvrant les portes des flux de conscience de Constance et de son futur amant: "Yet from his bowels, from his knees, from the middle of his breast he felt himself streaming towards her [...]. The desire he had was in his body, and his mind tried not to acquiesce. ${ }^{24}$ L'écrivain nous révèle 
les secrets de l'âme de ses personnages pour mieux nous faire saisir ce désir grandissant qui les envahit à toute allure, désir qui s'exprime dans leur for intérieur mais qui vient entièrement du corps, des membres, des organes et de la chair en quête de contact, bien que réprimés par l'esprit. Pascale Ferran détourne la nature purement verbale de cet extrait en inventant une suite de scènes vouées à signifier le désir de Constance pour Parkin d'abord, celui de Parkin pour Constance ensuite. Ayant obtenu un double de la clé du repaire du garde-chasse, et après y avoir passé quelques moments en sa compagnie, Constance se rend un jour à la clairière et, n'y trouvant personne, pénètre seule dans la cabane. Pascale Ferran, dans un élan de symbolisme qui n'a rien à envier à la prose imagée de l'écrivain, place alors un insert sur la clé de la jeune femme qui glisse dans la serrure. Dans la cabane, Constance trouve un gant sur une étagère. Le scénario dit : « Il est si vieux qu'il a gardé la forme de la main de celui qui l'a si souvent habité. Constance enfile le gant de cuir, comme sans y penser. $»^{25}$ Pascale Ferran s'est montrée si fidèle à son script que le gros plan sur la main de Constance enfilant le gant de Parkin, dont le cuir usé craque à son contact, se pare de la même symbolique que celui sur la clé. Constance habite le lieu de Parkin, son intimité, elle pénètre son monde et, à défaut de toucher directement cet homme, elle entre en contact avec lui par un transfert sur un simple objet, objet de désir, certes inanimé mais pourtant si plein de l'autre, portant trace, empreinte du vivant. Un fondu au noir raccorde avec un gros plan sur les mains de Constance, habillées des fameux gants de Parkin, ratissant la terre pour planter des graines devant la cabane du garde-chasse. Parkin, lui aussi surpris de voir sa "maîtresse » ainsi agenouillée pour travailler la terre, interrompt son travail un instant et la regarde. Puis ils se réunissent sur les marches de la cabane pour se rafraîchir. Parkin sert à boire à la jeune femme exténuée et la regarde à nouveau. Comme dans le roman, la caméra de Ferran adopte un temps le point de vue de l'homme et scrute le profil haletant de Constance en très gros plan et de trois-quarts dos via un raccord-regard intercesseur. Nous contemplons avec Parkin les gouttes de sueur qui perlent sur le front de la jeune femme et les petites mèches de cheveux collées à sa peau. En revenant sur le gardechasse, le montage achève de signifier le sentiment qui vient de naître en lui et le désir qui déjà l'envahit. C'est au tour de Parkin d'observer la jeune femme et de la toucher par un regard défiant les lois de l'optique, une vision tactile qui tend les corps l'un vers l'autre avant qu'ils ne s'embrassent réellement.

La révélation a eu lieu dans la séquence de la toilette, où l'idée nouvelle du corps a surgi ; puis une première approche, timorée et dérobée à notre regard, semble avoir été rendue possible dans le pré aux jonquilles; Constance est ensuite parvenue à un contact de substitution avec pour ligne de fuite le véritable contact charnel, la révolution des êtres par l'union corporelle - et, in fine, la métamorphose. Le premier rapport sexuel advient quand Parkin, après avoir mis un poussin nouveau-né dans les mains d'une Constance violemment émue par cette nouvelle vie palpitante, prend la femme dans ses bras pour la consoler. Brusquement emporté par le désir, Parkin laisse sa main glisser de l'épaule vers le sein - "the touch of hands and breasts, the touch of the whole body to body", comme l'annonçait Lawrence un peu plus tôt. Pascale Ferran fait un très gros plan sur la main de Parkin, se déplaçant avec elle dans un mouvement tumultueux du dos de Constance vers sa poitrine pour finalement la voir agripper le sein de la jeune femme à travers le velours pourpre de son tailleur. Le choix du costume et de sa matière n'est pas anodin, et Pascale Ferran filme cette séquence, comme la plupart de celles que nous avons évoquées, sans y appliquer de musique, pour laisser les bruits du monde s'exprimer et révéler les contacts 
de la peau avec la mousse d'un arbre, l'eau d'un ruisseau ou l'étoffe d'un tissu, jouant sur toute une synesthésie. L'écrivain insiste sur ce geste, sur ce toucher qui se réalise enfin :

His hand slid slowly round her body, touching the breasts that hung inside her dress [...] his fingers did not cease delicately touching her breasts. [...] His hand touching her breast was like flames. ${ }^{26}$

La chaleur du contact, qui est celle de l'émotion extrême des amants, n'a d'équivalent que la fébrilité de la caméra portée de Pascale Ferran, qui semble vibrer du même désir que les personnages et dont la spontanéité s'accorde à leur surprise. Les rapports charnels se succéderont ensuite et marqueront autant de nouvelles étapes dans une progression exponentielle vers le plaisir et la tendresse, jusqu'au couronnement amoureux des deux amants.

\section{NOTES}

1. Aussi connu sous le titre John Thomas and Lady Jane, et intitulé en français Lady Chatterley et l'homme des bois. Il s'agit de la deuxième version d'un roman réécrit trois fois par D. H. Lawrence (1885-1930). La troisième version de cette histoire, et la plus célèbre, Lady Chatterley's Lover ( L'amant de Lady Chatterley), a fait l'objet de multiples adaptations cinématographiques, la plus connue étant celle de Marc Allégret réalisée en 1955.

2. L'adaptation par Pascale Ferran a fait l'objet de deux versions distinctes, l'une pour la télévision, l'autre pour le cinéma, qui fut récompensée par le prix Louis-Delluc et obtint cinq César en 2007.

3. D. H. Lawrence, The Second Lady Chatterley's Lover, Oneworld Classics, Richmond, 2007, p. 99 ( «Ce dont son âme avait un besoin urgent, c'était d'une rémission, d'une espérance, d'un contact. Voilà ce qu'il lui fallait: ni quelque révélation, ni quelque idée nouvelle; mais un contact nouveau. Un contact seulement.", traduction de Jean Malignon, dans D. H. Lawrence, Lady Chatterley et l'homme des bois, $2^{\circ}$ version de L'Amant de Lady Chatterley, Paris, Gallimard, «Du monde entier ", 2006 [1977], p. 164. Désormais, les traductions fournies entre parenthèses seront tirées de la référence pré-citée).

4. Dans une lettre à Aldous Huxley, citée dans le dossier de presse du film Lady Chatterley, Ed. Ad Vitam, 2006, disponible en ligne : <http://medias.unifrance.org/medias/233/120/30953/presse/ lady-chatterley-2006-presskit-1.pdf> (consulté le 28/04/2013).

5. "Un conte filmé comme un documentaire. Entretien avec Pascale Ferran ", Les Cahiers du cinéma, $\mathrm{n}^{\circ} 617$, novembre 2006, p. 12.

6. Gilles Deleuze écrit : «On parlera d'haptique chaque fois qu'il n'y aura plus subordination étroite [entre la main et l'œil], ni subordination relâchée ou connexion virtuelle, mais quand la vue elle-même découvrira en soi une fonction de toucher qui lui est propre, et n'appartient qu'à elle, distincte de sa fonction optique. », dans Francis Bacon. Logique de la sensation, Paris, Ed. du Seuil, « L'ordre philosophique », 2002 [1981], p. 146.

7. D. H. Lawrence, The Second Lady Chatterley's Lover, op. cit., p. 41. (« Le silence l'assurait à ce point de sa solitude qu'elle se décida tout d'un coup à franchir la petite porte de la cour ; et là elle s'arrêta net, comme si elle venait de recevoir un coup de fusil. ", p. 75.)

8. D. H. Lawrence, The Second Lady Chatterley's Lover, op. cit., p. 41-42. (« Le torse blanc de l'homme lui était apparu, si beau : comme il s'épanouissait dans ce sombre décor ! Corps divin, ferme, 
ondoyant et soyeux, blanc. Telle l'arche blanche de la vie, il se tenait là, penché au-dessus de l'eau. Oui, tout cela était à ses yeux, et bien qu'elle s'en défende, de l'ordre du divin. Ainsi il existait encore un monde capable de resplendir de pureté et de puissance, puisque là, en ce terne après-midi, resplendissait sur tout le paysage, soyeuse et ferme, la peau de ce corps d'homme. [...] Elle venait de voir la beauté, et la beauté vivante. Ce corps appartenait au monde des dieux entrevu au milieu des ténèbres, comme une révélation. ", p. 75-76).

9. Si le sexe de l'être divin est inversé, son rôle reste le même: Diane et Parkin sont tous deux chasseurs.

10. D. H. Lawrence, The Second Lady Chatterley's Lover, op. cit., p. 42. («Un vaste apaisement l'envahissait, en même temps qu'un sentiment de vénération. Le sentiment subit de la pure beauté, d'une beauté devenue acte, vivante, avait restauré en ce cœur le besoin d'adorer. Non pas qu'elle eût adoré cet homme, le corps de cet homme ; mais la ferveur venait de monter en elle, parce qu'elle avait vu une chose de beauté pure, et qui était vivante, et qui l'avait atteinte jusqu'au plus vif d'elle-même. C'était comme si elle avait touché Dieu et qu'elle avait été rendue à la vie. Immense et resplendissante blancheur ! ", p. 76).

11. D. H. Lawrence, The Second Lady Chatterley's Lover, op. cit., p. 41 (« Il était si près de Constance qu'elle aurait pu le toucher. ", p. 75.)

12. Gilles Deleuze, Cinéma 2. L'Image-temps, Paris, Les Editions de Minuit, «Critique », 2002, p. 35.

13. D. H. Lawrence, The Second Lady Chatterley's Lover, op. cit., p. 41 («Sans faire de bruit, elle s'éclipsa parmi les arbres aux branches chargées de pluie. Elle avait hâte d'être hors de la vue de Parkin », p. 75.)

14. Notons que dans la version longue du film une troisième apparition survient, qui montre Parkin achevant sa toilette et s'essuyant avec une serviette, confirmant la thèse selon laquelle Constance ne peut pas être dans le simple ressouvenir d'images volées juste avant sa fuite.

15. Gaston Bachelard, L'Air et les songes, Essai sur l'imagination du mouvement, Le Livre de poche, «Biblio essais ", 2007, p. 217.

16. Gilles Deleuze, Cinéma 2. L'Image-temps, op. cit., p. 29.

17. Gilles Deleuze, ibid., p. 9.

18. Lorand Gaspar dans la préface à D. H. Lawrence, Poèmes, Paris, Gallimard, « Poésie », 2007, p. 13.

19. Pascal Quignard, la Nuit sexuelle, Paris, J'ai lu, «En images », 2009, p. 161.

20. On pense alors aux leçons de Robert Bresson : «Ne cours pas après la poésie. Elle pénètre toute seule par les jointures (ellipses) », ou «Montage. Passage d'images mortes à des images vivantes. Tout refleurit.», dans Robert Bresson, Notes sur le cinématographe, Paris, Gallimard, «Folio », p. 39 et p. 89.

21. D. H. Lawrence, The Second Lady Chatterley's Lover, op.cit., p. 99 (" Mais par la douceur, par le renoncement à toute affirmation de soi, par la plénitude de notre moi véritable et profond, nous pouvons nous rapprocher d'un autre humain et connaître ainsi le meilleur et le plus délicat de la vie : le contact. Contact des pieds sur le sol, contact des doigts sur un arbre, sur un être vivant. Contact des mains et des seins. Contact de tout ce corps et d'un autre corps; mutuelle pénétration de l'amour passionné. Voilà la vie. Et c'est par le contact que nous vivrons, tous, autant que nous sommes ", p. 165).

22. D. H. Lawrence, The Second Lady Chatterley's Lover, op. cit., p. 78 («[...] un jeune pin qui se balançait un peu. C'était comme une créature animée qui se frottait doucement contre elle ; une grande chose vivante, avec son extrémité, là-haut, flottant au vent ! », p. 133).

23. Pascale Ferran et Roger Bohbot, Lady Chatterley et l'homme des bois, Scénario d'après l'œuvre de D. H. Lawrence, Paris, Gallimard, 2008, p. 65 (supplément au DVD Lady Chatterley et l'homme des bois, Arte Vidéo, Edition Prestige, 2008). 
24. D. H. Lawrence, The Second Lady Chatterley's Lover, op. cit., p. 103 (« Or il se sentait venir à elle, avec toutes ses viscères, avec ses genoux, avec le souffle de ses poumons [...]. Son désir était logé dans la chair, et l'esprit s'efforçait de ne pas donner son accord », p. 170).

25. Pascale Ferran et Roger Bohbot, Lady Chatterley et l'homme des bois, Scénario d'après l'œuvre de D. H. Lawrence, op. cit., p. 65.

26. D. H. Lawrence, The Second Lady Chatterley's Lover, op. cit., p. 105 (« La main contournait lentement son corps, touchant ses seins enfermés dans sa robe [...] sans cesser de lui toucher des doigts délicatement les deux seins. [...] Cette main sur sa poitrine ! C'était de la flamme!», p. 173).

\section{RÉSUMÉS}

The Second Lady Chatterley's Lover (1927) de David Herbert Lawrence, adapté au cinéma en 2006 par la cinéaste française Pascale Ferran (Lady Chatterley), fait le récit d'une renaissance, d'un épanouissement corporel, d'une réincarnation de la chair. Le romancier, et la cinéaste à sa suite, traitent ce lent apprentissage du contact physique en convoquant une expérience tactile du monde. Évoquant une "vision haptique » telle que définie par Gilles Deleuze, le regard se fait l'agent du contact entre deux êtres et rompt les lois cartésiennes de l'espace pour invoquer la figure de l'autre et la rendre quasi-palpable. S'opère ensuite un déplacement vers un toucher absent, quand le contact est transféré sur des objets vivants ou portant trace du vivant, dans un rapprochement où la figure de la main tient une place essentielle - autant de passages obligés avant que l'étreinte espérée ne soit enfin célébrée et ne conduise au couronnement amoureux des amants.

Lady Chatterley - from novel to film : regaining the body through the sense of touch David Herbert Lawrence's The Second Lady Chatterley's Lover (1927) was adapted for film in 2006 by french filmmaker Pascale Ferran (under the title Lady Chatterley). It tells the tale of a resurgence, a corporal blossoming, a reincarnation of the flesh. The filmmaker, in the wake of the novelist, deals with this slow learning of the physical contact through the invocation of a tactile experience of the world. What french philosopher Gilles Deleuze defined as haptic vision is brought to mind when the look makes itself a cause for contact between two beings and breaks with the rational laws of space so as to call upon the figure of the other and make it almost tangible. A transfer towards an absent touch then occurs, when contact shifts to living objects, or some that bear a trace of life. In this new closeness, the figure of the hand holds an essential place - as many a prerequisite before the anticipated embrace is finally celebrated and leads to the lovers' romantic coronation.

\section{AUTEUR}

\section{RÉMI GONZALEZ}

Doctorant en Lettres Modernes à l'Université du Mirail (Toulouse II), prépare une thèse sur l'écriture cinématographique dans le roman français contemporain. Travaillant sur les liens entre littérature et cinéma, il a rédigé des mémoires universitaires sur l'adaptation 
cinématographique Lady Chatterley et sur L'Incident de Christian Gailly, adapté au cinéma par Alain Resnais. Co-auteur d'une intervention sur Laurent Mauvignier publiée aux presses universitaires de Dijon en mai 2012, il collabore régulièrement à la revue Éclipses (Douglas Sirk, Sidney Lumet, Jerzy Skolimowski, Arnaud Desplechin). 